\title{
DETERMINAÇÃO DA FERTILIDADE DO SOLO EM AREAS DE NASCENTES COM OCORRENCIA DE VOÇOROCA NO MUNICIPIO DE BRASIL NOVO, PARÁ
}

\section{Samia Cristina de Lima Lisboa ${ }^{1,}$ Sandra Andréa Santos da Silva ${ }^{2}$, Rainerio Meireles da Silva ${ }^{3,}$ José Farias Costa ${ }^{4}$, Lucimara dos Santos Lima ${ }^{5}$. \\ ${ }^{1}$ Discente do Curso de Engenharia Florestal da Universidade Federal do Pará, Campus Altamira, samiallisboa@gmail.com \\ ${ }^{2}$ Professora Doutora Adjunto IV do Curso de Engenharia Agronômica da Universidade Federal do Pará, Campus Altamira \\ ${ }^{3}$ Rainério Meireles da Silva, Professor Doutor Associado IV do Curso de Engenharia Agronômica da Universidade Federal do Pará, Campus de Altamira \\ ${ }^{4}$ Jose Farias Costa, Engenheiro Agrônomo, mestrando na Universidade Federal do Pará, Campus de Altamira \\ ${ }^{5}$ Lucimara dos Santos Lima, Engenheira Agrônoma da Universidade Federal do Pará, Campus de Altamira.}

\section{Recebido em: 22/09/2018 - Aprovado em: 23/11/2018 - Publicado em: 03/12/2018} DOI: $10.18677 /$ EnciBio_2018B35

\section{RESUMO}

O solo é um dos recursos que mais sofrem impactos e degradação, pois, o manejo inadequado desse recurso, acarreta sérios problemas ambientais, além de alterar fortemente os atributos do mesmo. Os processos erosivos comprometem diretamente a qualidade do solo. As maiores proporções erosivas no solo são as voçorocas, que surgem devido a retirada ou a diminuição da vegetação principal responsável pela proteção desse recurso. Esta pesquisa visa realizar um diagnóstico dos parâmetros químicos em uma voçoroca sob Área de Preservação Permanente, localizada no município de Brasil Novo - Pará. O estudo foi realizado a $40 \mathrm{~km}$ do município Altamira. Em transecto, determinou-se dois pontos de coleta (A e B), sendo A, o ponto mais alto da voçoroca e $B$ a área mais baixa do terreno, levando-se em consideração a topografia e as características superficiais do solo. De acordo com valores de referencias, observou-se que os teores dos atributos químicos não estão em níveis satisfatórios. Houve redução de $\mathrm{Ca}, \mathrm{Mg}, \mathrm{K}, \mathrm{P}$ e teores de matéria orgânica, além do acréscimo dos teores de $\mathrm{Al}, \mathrm{H}+\mathrm{Al}$ e saturação por alumínio ( $m \%$ ) no ambiente estudado. Portanto, é indispensável a realização de uma intervenção antrópica para estagnar o desenvolvimento da erosão, pois, a área mostra-se fragilizada.

PALAVRAS-CHAVE: área de preservação permanente, atributos químicos, erosão.

\section{DETERMINATION OF SOIL FERTILITY IN AREAS OF BIRTHS WITH OCCURRENCE OF VOÇOROCA IN THE MUNICIPALITY OF BRAZIL NOVO - PARÁ-BRAZIL}

\begin{abstract}
Soil is one of the resources that suffer the most impacts and degradation, since the inadequate management of this resource causes serious environmental problems, as well as greatly altering its attributes. Erosive processes directly compromise soil quality. The highest erosion rates in the soil are the gullies, which arise due to the
\end{abstract}


removal or reduction of the main vegetation responsible for the protection of this resource. This research aims to perform a diagnosis of chemical parameters in a gull under Permanent Preservation Area, located in the municipality of Brasil Novo Pará. The study was carried out $40 \mathrm{~km}$ from Altamira municipality. In transect, two collection points ( $A$ and $B$ ) were determined, with $A$ being the highest point of the voçoroca and $B$ the lowest area of the terrain, taking into account the topography and the surface characteristics of the soil. According to reference values, it was observed that the contents of the chemical attributes are not at satisfactory levels. there was reduction of $\mathrm{Ca}, \mathrm{Mg}, \mathrm{K}, \mathrm{P}$ and organic matter contents, as well as the increase of $\mathrm{Al}, \mathrm{H}+\mathrm{Al}$ and saturation by aluminum $(\mathrm{m} \%)$ in the studied environment. Therefore, it is indispensable to carry out anthropic intervention to stagnate the development of erosion, because the area is fragile.

KEYWORDS: permanent preservation area, chemical attributes, erosion.

\section{INTRODUÇÃO}

A preocupação com a qualidade ambiental e a recuperação de áreas degradadas tem sido destaque para muitos estudos e pesquisas. O solo é um dos recursos que mais sofrem impactos, pois, o processo de desertificação, o desmatamento e o manejo inadequado, são fatores que acarretam sérios problemas ambientais, além de alterar fortemente os atributos, tanto físicos quanto químicos, sendo o físico o primeiro a responder essas perturbações (AMARAL et al., 2013). Tais fatores levam a degradação de grandes áreas e essas degradações vêm se expandindo de forma acelerada, comprometendo diretamente a qualidade do solo.

A erosão é um dos processos naturais fundamentais na formação de solos, mas devido as interferências antrópicas, vem ganhando proporções maiores que o natural, direcionando para grandes transtornos, devido a movimentação e deslizamento de terra (BARBOSA et al., 2014). Quando essas erosões atingem uma grande profundidade dar-se origem as voçorocas causadas pelo impacto da chuva e demais intempéries, impactos esses indiciados principalmente pela retirada da cobertura vegetal do solo, pois, quando o mesmo fica descoberto a superfície fica exposta ao sol e principalmente às chuvas, tornando-se suscetível de carregamento por enxurradas, causando desta forma, o início de um processo de degradação no solo (COSTA et al., 2015).

Quando se trata de uma área de preservação permanente, a preocupação é ainda maior, pois o processo de degradação não só ameaça a fertilidade do solo, como também a qualidade da água, pois os processos erosivos podem apresentar maior proporção nessas áreas e modificam consideravelmente a dinâmica hidrológica (COUTINHO, 2013). Assim a preocupação com a qualidade ambiental de uma propriedade no $\mathrm{km} \mathrm{40,} \mathrm{no} \mathrm{município} \mathrm{de} \mathrm{Brasil} \mathrm{novo} \mathrm{-} \mathrm{Pará,} \mathrm{que}$ atualmente se encontra afetada por voçoroca, merece especial atenção, uma vez que a mesma se encontra em um local com presença de nascente e o processo de assoreamento está em estágio avançado.

Sabendo que os atributos químicos variam de acordo com o tipo de solo e exercem diferentes influências sobre a erosão (BERTONI; LOMARDI NETO, 2014) a avaliação desses atributos em áreas com voçorocas, permite monitorar a sensibilidade da mesma às ações humanas, fornecendo subsídios para o manejo adequado e racional deste recurso.

Posto isto, esta pesquisa visou realizar diagnóstico dos parâmetros químicos do solo de modo a contribuir com informações iniciais das áreas alteradas através do conhecimento prévio do nível de fertilidade do solo, além de fornecer 
informações que venham a permitir a recomendação de espécies futuras de modo a proporcionar a regeneração da área de preservação permanente e assim minimizando os processos de erosão e qualidade do recurso hídrico.

\section{MATERIAL E MÉTODOS}

O estudo foi realizado em uma voçoroca no $40 \mathrm{~km}$ do município de Brasil Novo, estado do Pará. De acordo com dados do Plano Municipal de Meio Ambiente (PMMA BRASIL NOVO, 2017) do município, a vegetação do município é heterogênea, formada por campos, capoeiras e mata secundária. Quanto ao clima à temperatura média anual na região é de $27,2^{\circ} \mathrm{C}$, valor definido como a média das temperaturas medias anuais dos postos analisados, em Porto de Moz e Altamira, uma vez que não existem postos de observação em Brasil Novo, e a precipitação média anual é de $1.680 \mathrm{~mm}$. Os meses mais chuvosos vão de dezembro a maio e os menos chuvosos, de junho a novembro.

As amostras de solo foram coletadas no mês de janeiro de 2018 (período chuvoso), de acordo com a metodologia proposta por Santos et al. (2015). A metodologia consiste na coleta de amostras compostas deformadas, as quais foram coletadas com auxílio do trado holandês com determinação da profundidade desejada de $0-20 \mathrm{~cm}$. A área correspondente ao estudo foi dividida em unidades homogêneas, as quais foram determinadas em um transecto dois pontos de coleta ( $A$ e $B$ ), sendo $A$, no ponto mais alto da voçoroca e $B$ a área mais baixa, levando-se em consideração a topografia do terreno e as características superficiais do solo (Figura 1).

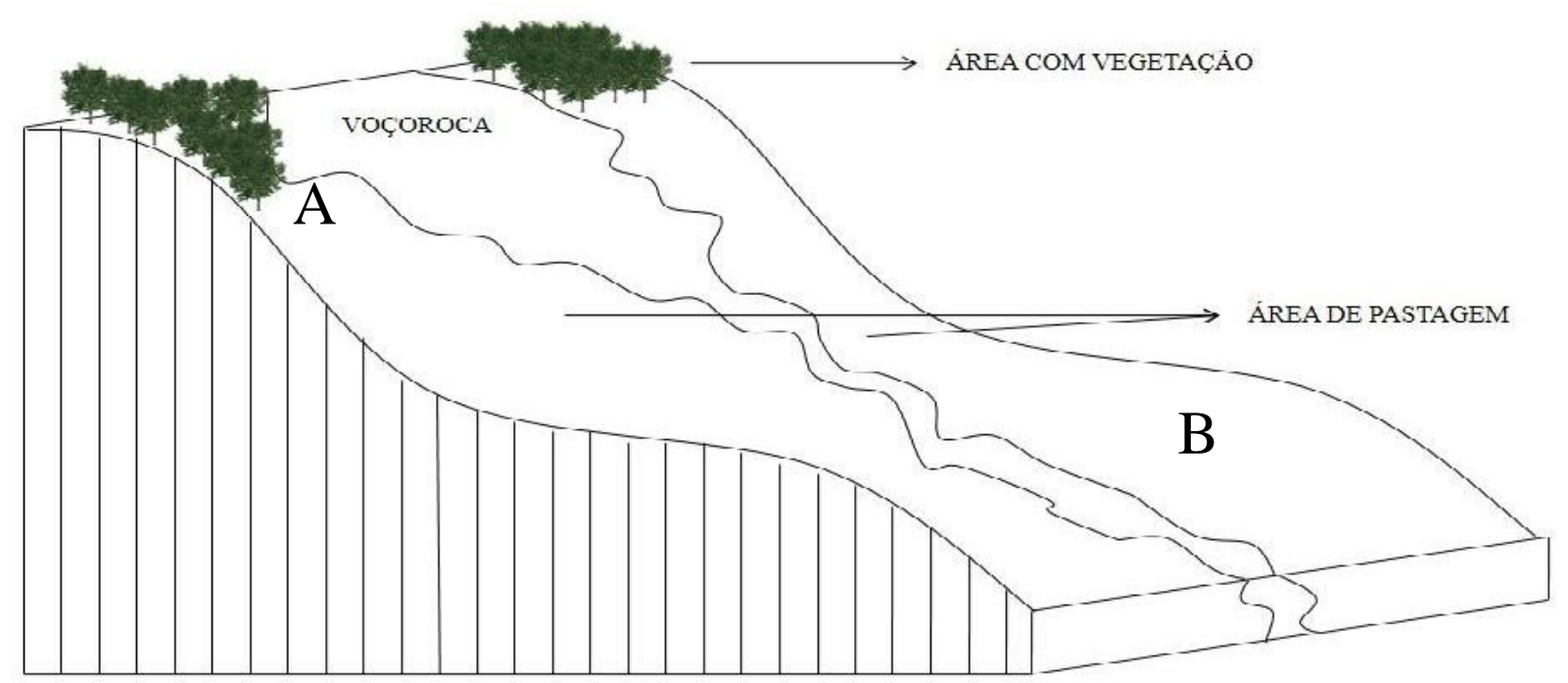

FIGURA 1: Croqui da área de estudo (Fonte: Silva, 2018).

O estudo foi realizado com duas repetições, sendo cada formada por uma amostra, totalizando duas amostras por tratamento. As amostras deformadas foram submetidas ao preparo da Terra Fina Seca ao Ar (TFSA), no laboratório de Solos da Universidade Federal do Pará, Campus de Altamira - UFPA/CALT e posteriormente encaminhadas para análise química no laboratório da Universidade Federal Rural (UFRA), campus de Belém.

As análises realizadas compreenderam nas determinações de: $\mathrm{pH}$, matéria orgânica $(\mathrm{MO})$, fósforo $(\mathrm{P})$, potássio $(\mathrm{K}+)$, cálcio $(\mathrm{Ca})$, magnésio $(\mathrm{Mg})$, alumínio (Al), acidez potencial $(\mathrm{H}+\mathrm{Al})$, soma de bases trocáveis $(\mathrm{SB})$, capacidade de troca catiônica (CTC), saturação por alumínio (M\%) e a saturação por base (V\%). 
Os métodos empregados foram os preconizados de acordo a Embrapa (2009) e as interpretações dos resultados foram baseadas nos valores de referência de EMBRAPA (2017).

A análise estatística foi realizada através do teste de Wilcoxon (MannWhitney e Wilcoxon, $P<0,05)$, técnica não paramétrica extremamente interessante para análises de dados qualitativos dos dois tratamentos relacionados. $O$ teste foi interpretado através do software Excel 2013.

A comparação foi realizada entre Ponto A e B, em seguida os valores entre os tratamentos foram subtraídos e submetidos ao teste dos sinais para avaliar em quais dos tratamentos, os parâmetros químicos apresentaram melhores valores. Avaliou-se os resultados da comparação entre os pontos na tabela de amostras de Wilcoxon para saber se houve diferença significativa ou não.

Uma vez que os resultados obtidos para o teste t de Wilcoxon em relação aos valores mínimo e máximo obtidos dos parâmetros avaliados não estiverem dentro do intervalo de tabulação dos graus de liberdade, a hipótese de nulidade (H0) é rejeitada e, consequentemente, a hipótese alternativa (Ha) é aceita, a uma margem de $0,05 \%$ de probabilidade de erro.

\section{RESULTADOS E DISCUSSÃO}

Os resultados obtidos através da análise química para as variáveis avaliadas nesta pesquisa estão descritos na Tabela 1.

TABELA 1 Teores médios dos atributos químicos de solo dois pontos distintos da voçoroca no município de Brasil Novo - PA.

\begin{tabular}{|l|l|l|l|l|l|l|l|l|l|l|l|l|}
\hline Área & $\mathbf{P}$ & $\mathbf{K}$ & $\mathbf{C a}$ & $\mathbf{M g}$ & $\mathbf{A l}$ & $\mathbf{H + A l}$ & $\mathbf{P . H}$ & $\mathbf{M . O}$ & $\mathbf{S B}$ & $\begin{array}{l}\mathbf{C T C} \\
\mathbf{( T )}\end{array}$ & $\mathbf{m} \%$ & $\mathbf{V} \%$ \\
\hline & $\mathbf{m g} / \mathbf{d m}^{\mathbf{3}}$ & \multicolumn{3}{|c|}{$\mathbf{c m o l}_{\mathbf{c}} \mathbf{d d m}^{\mathbf{3}}$} & & $\mathbf{H}_{\mathbf{2}} \mathbf{0}$ & $\mathbf{m g} / \mathbf{d m}^{\mathbf{3}}$ & & & & \\
\hline A & 4,55 & 0,05 & 1,15 & 0,45 & 0,64 & 4,01 & 4,25 & 1,92 & 1,65 & 6,46 & 29,75 & 28,53 \\
\hline B & 2,9 & 0,35 & 0,80 & 0,60 & 0,79 & 3,9 & 4,45 & 1,37 & 2,38 & 6,29 & 24,75 & 38,07 \\
\hline
\end{tabular}

T1=Área alta da voçoroca; T2 = Área baixa da voçoroca.

Para interpretação dos dados acima, foi utilizado os valores de referência da Embrapa (2010), para classificação dos teores em níveis baixos, médios e altos. Deste modo, o valor de $\mathrm{pH}$, caracteriza o solo com acidez elevada (alta), com valores entre 4,25 e 4,45 para o ponto $A$ e $B$, respectivamente, pois a faixa ideal de pH segundo Embrapa (2010), para a maioria dos vegetais está entre 5,5 a 6,5.

Valores semelhantes foram encontrados no estudo de Voçorocas no município de Brasil Novo por Souza et al. (2018), onde foram obtidos valores de pH entre 4,60 a 4,95 em quatro pontos distintos próximos a erosão, tal resultado implicou na baixa fertilidade do solo, caracterizando assim um solo extremamente pobre, em que o seu potencial nutricional está bastante reduzido. $\mathrm{O} \mathrm{pH}$ tem efeito direto sobre a disponibilidade dos nutrientes essenciais às plantas, na atividade dos microrganismos e da matéria orgânica, sendo por isso importante sua monitoração.

O potássio depois do nitrogênio, é o nutriente mineral mais requerido em quantidade pelas espécies vegetais, devido a mobilidade que o mesmo faz nas plantas, além de ser responsável pelas trocas gasosas. Quando este apresentar teor inferior à $0,156 \mathrm{cmol}_{\mathrm{c} .} \mathrm{dm}^{-3}$ são considerados baixos para diversas culturas. 
Entre os pontos estudados, ponto B apresentou teor médio, uma vez que a faixa de potássio está entre 0,156 a $0,39 \mathrm{cmol}_{\mathrm{c} . \mathrm{dm}^{-3}}$. O valor neste ponto pode ser explicado pela ação da água das chuvas pois, independentemente da decomposição da matéria orgânica, pode constituir um fator importante na lixiviação de nutrientes de restos vegetais.

Os teores de cálcio $(\mathrm{Ca})$ foram baixos nos dois pontos estudados, sendo 1,15 e $0,8 \mathrm{cmol}_{\mathrm{c} .} \mathrm{dm}^{-3}$, para os pontos $A$ e $B$, respectivamente, estes valores são inferiores aos de referência de $1,5 \mathrm{cmol}_{\mathrm{c}} \mathrm{dm}^{-3}$. Quanto aos valores de magnésio $(\mathrm{Mg})$ e fósforo $(\mathrm{P})$, os pontos estudados apresentaram valores baixos, inferiores à $0,6 \mathrm{cmol}_{\mathrm{c} . \mathrm{dm}^{-3}}$ e $5 \mathrm{mg} / \mathrm{dm}^{3}$, respectivamente.

Os valores expressos são decorrentes da erosão hídrica presente na área o que provoca o decréscimo na fertilidade do solo, influenciando na dificuldade da regeneração natural. Os estudos de Centeno et al. (2017), corroboram com o resultado, quando o mesmo afirma que geralmente os teores de $\mathrm{P}$ apresentou nível baixo quando se trata de solos com características arenosas, pois tal nutriente é fácil de ser perdido devido o processo rápido de lixiviação em solos com essa textura.A redução dos nutrientes acima, quando se trata de ambientes de voçorocas, pode estar relacionada diretamente ao conteúdo de matéria orgânica condicionada pela remoção de vegetação nesse ambiente, determinando menor ciclagem de nutrientes e aumento de perdas por lixiviação (GOMIDE et al., 2010). A matéria orgânica (MO) apresentou-se com teores considerados de baixo a médio, com valores de 1,37 e 1,92 g. $\mathrm{kg}^{-1}$, destaque para a área A que apresentou teores médios de $\mathrm{MO}$ de 1,92 g. $\mathrm{kg}^{-1}$. Para Embrapa (2010), o valor 1,6 a 3,0 g. $\mathrm{Kg}^{-1}$, são considerados teores médios.

Valores de 1,35 g. $\mathrm{kg}^{-1}$, foi encontrado por Souza et. al (2018), representando maior teor de matéria orgânica em área de voçoroca no município de Brasil Novo na camada superficial, onde o mesmo relacionou a quantificação MO da voçoroca a presença de pastagens, que pode estar proporcionando um acúmulo, podendo ser um indicador deste resultado. Dernadin et al. (2014), afirma que a sustentação da cobertura vegetal do tipo floresta garante uma maior contribuição de resíduos tanto na camada superficial e subsuperficial do solo. Tendo em vista que o ponto A se encontra próxima à área de encosta, pressupõe que o maior teor de matéria orgânica esteja ligado ao tipo de vegetação encontrada no ponto de coleta.

TABELA 2 -Teores médios dos atributos químicos de solo em dois pontos distintos da voçoroca no município de Brasil Novo - PA

\begin{tabular}{|l|c|c|c|c|c|c|c|c|c|c|c|c|}
\hline Área & $\mathbf{P}$ & $\mathrm{K}$ & $\mathbf{C a}$ & $\mathbf{M g}$ & $\mathbf{A l}$ & $\mathbf{H}+\mathbf{A l}$ & $\mathbf{P . H}$ & $\mathbf{M . O}$ & $\mathbf{S B}$ & $\mathbf{C T C}(\mathbf{T})$ & $\mathbf{m} \%$ & $\mathbf{V} \%$ \\
\hline & $\mathbf{m g} / \mathbf{d m}^{\mathbf{3}}$ & \multicolumn{3}{|c|}{$\mathbf{c m o l}_{\mathbf{c}} / \mathbf{d m}^{\mathbf{3}}$} & & $\mathbf{H}_{\mathbf{2}} \mathbf{0}$ & $\mathbf{m g} / \mathbf{d m}^{\mathbf{3}}$ & & & & \\
\hline A & $\mathrm{B}$ & $\mathrm{B}$ & $\mathrm{B}$ & $\mathrm{A}$ & $\mathrm{M}$ & $\mathrm{M}$ & $\mathrm{A}$ & $\mathrm{M}$ & $\mathrm{B}$ & $\mathrm{M}$ & $\mathrm{M}$ & $\mathrm{M}$ \\
\hline $\mathrm{B}$ & $\mathrm{B}$ & $\mathrm{M}$ & $\mathrm{B}$ & $\mathrm{M}$ & $\mathrm{M}$ & $\mathrm{M}$ & $\mathrm{A}$ & $\mathrm{B}$ & $\mathrm{M}$ & $\mathrm{M}$ & $\mathrm{M}$ & $\mathrm{M}$ \\
\hline
\end{tabular}

$\mathbf{B}=$ Baixo, $\mathbf{M}=$ Médio, $\mathbf{A}=$ Alto

A saturação por Base (V\%) está relacionada diretamente à fertilidade natural do solo, considerando-o como Eutrófico (alta fertilidade) quando V\% estiver acima de $50 \%$, e distróficos (Baixa fertilidade) com índices do V\% menores que 50\% (SILVA et., al 2015). Os valores do V\% das áreas avaliadas apresentaram $28,53 \%$ para o ponto $A$ e $38,07 \%$ para o ponto $B$, implicando assim em baixa saturação, ou seja, abaixo do valor recomendado, caracterizando desta forma um solo distrófico, com baixo potencial de fertilidade. Tais valores corroboram com Oliveira et al. (2018), no estudo realizado em áreas de voçorocas no município de Brasil Novo, 
que apresentou valores entre $21,3 \%$ e $33,17 \%$ para as áreas de capoeira e vegetação, respectivamente, apresentando a classificação também de solos distróficos.

A soma de bases (SB) é dada pelos teores de $\mathrm{Ca}, \mathrm{Mg}$ e $\mathrm{K}$, o mesmo apresentou nível baixo no ponto A. Para Ronquim (2010), a soma de bases apresenta menor valor onde $\mathrm{pH}$ é ácido, tal afirmativa é válida para esse estudo, visto que a maior acidez também foi indicada no mesmo ponto. Os resultados corroboram com os encontrados por Oliveira et al. (2017), em estudo de áreas de capoeira e pastagem em São Domingos do Araguaia- PA, que apresentaram valores de SB baixos em áreas de pastagens na região amazônica, que segundo os autores o valor se deve ao baixo teor de $\mathrm{MO}$ encontrada no solo, sendo um fator limitante na ciclagem de nutrientes.

Quanto à acidez potencial $(\mathrm{H}+\mathrm{Al})$, Al e saturação por alumínio (m\%), os mesmos apresentaram-se com valores médios, o que torna o solo limitante para 0 desenvolvimento de vegetação, uma vez que o teor de alumínio deve ser mínimo para evitar prejuízos às raízes das plantas, além de reduzir a disponibilidade de $P, S$ e cátions (BRAGA, 2012). Segundo Gomide et al. (2011), esses resultados podem também estar relacionados a baixa concentração de $\mathrm{MO}$ encontrada na localidade, sendo um aspecto que possivelmente possa estar afetando de maneira considerável o processo de ciclagem de nutrientes.

A CTC, que está diretamente relacionada ao teor de matéria orgânica no solo (YADA, 2015). Este atributo apresentou teores abaixo do ideal de $10 \mathrm{cmol}_{\mathrm{c}} \mathrm{dm}^{3}$ nos dois pontos avaliados, sendo que a área $B$ foi a que apresentou maior valor, com 6,43 $\mathrm{cmol}_{\mathrm{c} .} \mathrm{dm}^{-3} \mathrm{~cm}$, aproximando-se a área A que apresentou CTC de 6,29 $\mathrm{cmol}_{\mathrm{c}} \cdot \mathrm{dm}^{-3}$. O baixo valor de CTC do solo estudado se dá devido a textura do solo caracterizada como arenosa pois, a importância da CTC está relacionada diretamente com a estruturação e consistência dos solo, correspondendo à soma das cargas negativas nas partículas microscópicas do solo: fração argila e matéria orgânica (CARVALHO, 2018), ou seja, quanto maior for o teor de argila maior será o valor da CTC.

De modo geral, observou-se que o solo apresenta características de degradação semelhantes tanto na parte mais alta como na parte mais baixa da voçoroca, mostrando de certa forma, uma fertilidade bastante comprometida. $\mathrm{Na}$ Tabela 3, constam os resultados do teste de Wilcoxon $(P<0,05)$, que apresenta as comparações das amostras de solo entre os pontos A e B da voçoroca, nas profundidades de 0 a $20 \mathrm{~cm}$.

TABELA 3 - Comparação dos atributos químicos das amostras de solo entre as unidades amostrais da voçoroca por meio da aplicação do teste t de Wilcoxon $(P<0,05)$.

\begin{tabular}{ccccc}
\hline Profundidade & $\begin{array}{c}\text { Tratamentos } \\
\text { Comparados }\end{array}$ & $\begin{array}{c}\text { Valor } \\
\text { Mínimo }\end{array}$ & Valor Máximo & Hipótese \\
\hline $\mathbf{0 - 2 0} \mathbf{c m}$ & $\mathrm{A}$ X B & 8 & 47 & Aceita HO \\
\hline
\end{tabular}

De acordo com a tabela 3, na aplicação estatística os tratamentos apresentaram similaridades em seus parâmetros químicos, apresentando a hipótese de nulidade $(\mathrm{H} 0)$. A hipótese foi aceita pois os parâmetros avaliados estão dentro do intervalo de tabulação de dados dos graus de liberdade, onde o valor mínimo é 8 e o valor máximo e 47, ou seja, não houve diferença significativa de acordo com a tabela 
de Wilcoxon para pequenas amostras. Apesar dos valores da análise química serem diferentes nos tratamentos, a variação é mínima, visto que a área estudada, ainda se encontra em fase inicial de erosão, logo, são poucos os atributos alterados de um ponto para outro. O mesmo método estatístico foi aplicado por Oliveira et al. (2018) e Souza et al. (2018) para comparação entre tratamento também em estudos de voçorocas no município de Brasil Novo-Pará.

\section{CONCLUSÃO}

O surgimento das voçorocas na região está diretamente interligado as características do relevo (declividade e comprimento da encosta), ocorrência de processos erosivos (erosão hídrica) e a influência das atividades humanas (desmatamentos e queimadas). Assim, o estudo permitiu concluir que a região analisada necessita de intervenções antrópicas através da aplicação de manejo do solo na área, para que ocorra a minimização do avanço da erosão, pois a mesma mostra-se fragilizada comprometendo a qualidade ambiental do referido ecossistema.

Os dados estudados nesta pesquisa, subsidiam informações necessárias para que ocorra a revegetação da área, respeitando as exigências nutricionais das espécies a serem incorporadas.

\section{REFERÊNCIAS}

AMARAL, A. J. COGO, N. P.; BERTOL, I.; SANTOS, P. G.; WERNER, R. S. Erosão hídrica e escoamento superficial em função de tipos e doses de resíduo cultural em dois modos de semeadura direta. Revistas de Ciências Agroveterinarias, Lages, 2013. Disponível em: <http://revistas.bvs-vet.org.br/rca/article/view/34862>. Acessado em: 05 jan. 2018.

BARBOSA, A. B.; ANDRADE, R. B.; MAGAGUTH, L.; NEGREIROS, A. B.; PEREIRA, G. Evolução de um voçorocamento em área urbana no munícipio de Cruzilia, MG. TerritoriumTerram, v.2, n.4, p.125-142, 2014. Disponível em: < http://seer.ufsj.edu.br/index.php/territorium_terram/article/view/799>. Acessado em: 14 jan. 2018.

BRAGA, G. N.; Alumínio, o Inimigo das Plantas. 2012. Disponível em: https://agronomiacomgismonti.blogspot.com/2012.html. Acessado em: 25 de Jul 2018.

BERTONI, L; NETO, L.F. Conservação do solo. São Paulo: Ícone. 2014. 355p.

CARVALHO, J. P. A capacidade de troca de cátions e o manejo de solos. 2018. Disponível em: http://www.pedologiafacil.com.br. Acessado em: 10 jul. 2018.

CENTENO, L. N.; GUEVARA, M. D. F.; CECCONELLO, S. T.; SOUSA, R. O. D.; TIMM, L. C. Textura do solo: Conceitos e aplicações em solos arenosos. Revista Brasileira de Engenharia e Sustentabilidade, Pelotas, v.4, n.1, p. 31-37, jun. 2017 
COSTA, C. D. O.; ALVES, M, C.; SOUSA, A. P.; SILVA, H. R. Propriedades químicas dos solos de uma sub-bacia hidrográfica sob processo de degradação ambiental. Revista Ciências Ambientais, Canoas, v.9, n.2, p. 37-50, 2015.

COUTINHO, L. M; ZANETTI, S. S; CECÍLIO, R. A; GARCIA, G. O, XAVIER, A. C. Usos da Terra e Áreas de Preservação Permanente (APP) na Bacia do Rio da Prata, Castelo-ES. Floresta e Ambiente, 2013, p. 434.

EMBRAPA - Empresa Brasileira de Pesquisa Agropecuária. Manual de análises químicas de solos, plantas e fertilizantes. 2. Ed. revista ampliada. Brasília, DF: Embrapa Informação Tecnológica, 2009, 627p.

EMBRAPA - Empresa Brasileira de Pesquisa Agropecuária. Recomendação de adubação e calagem para o estado do Pará. 2. Ed. revista ampliada. Brasília, DF: Embrapa Informação Tecnológica, 2010, 262p.

EMBRAPA - Empresa Brasileira de Pesquisa Agropecuária. Manual de métodos e analise do Solo. Teixeira, P. C... $3^{\circ}$ ed. revista e ampliada. Brasília, DF: Embrapa Informação Tecnológica, 2017, 574p.

ESCOBAR, F. B.; PIMENTEL, R. L.; TELLO, J. C. R. Avaliação da degradação de uma área da usina de Balbina como subsídio para recuperação e conservação ambiental, na Amazônia. Caminhos de Geografia, Uberlândia, 2017.

OLIVEIRA, A. N.; OLIVEIRA, A. N.; SILVA, K. R.; SILVA, J. A.; MELLO, A. H. Atributos químicos de solos sob diferentes sistemas de uso e manejo no Projeto de Assentamento Veneza- São Domingos do Araguaia. Agroecossistemas, v.9, n.1, p.170- 179, 2017.

OLIVEIRA, W. S., SILVA, S. A. S. SILVA, R. M. MORAIS, P. L. Determinação das propriedades químicas e físicas do solo em Áreas de Preservação Permamente (APP) com ocorrência de voçoroca, no município de Brasil Novo Pará. Revista Tree dimensional, Pro Floresta - Goiânia, v.3 n.5; p. 52. 2018.

Prefeitura Municipal de Brasil Novo-Pará. Secretaria municipal de meio ambiente. PLANO MUNICIPAL DE MEIO AMBIENTE DE BRASIL NOVO-PA. 2013.

RONQUIM, Carlos César. Conceitos de fertilidade do solo e manejo adequado para as regiões tropicais. Campinas, São Paulo, 2014.

SANTOS, R. D.; LEMOS, R. C.; SANTOS, H. G.; KER, J. C.; ANJOS, L. H. C. SHIMIZU, S. H. Manual de descrição e coleta de solo no campo. Revista ampliada, Viçosa, Sociedade Brasileira de Ciência do Solo, 2015, 100 p.

SANTOS, F. A. S., PIERANGELI, M. A. P., SILVA, F. L., SERAFIM, M. E., SOUZA, C. A. de. Atributos químicos, físicos e estoque de cálcio, magnésio, potássio e fósforo em solos de campos de murundus no Brasil. Revista Acta Amazônica, Cuiabá, 2015. p.101-110. 
SILVA, A.F. da, PEREIRA, M. J., ZIMBACK, C. R. L., LANDIM, P. M. B., SOARES, A., Simulação sequencial de atributos diagnósticos do solo. Revista Brasileira de Engenharia Agrícola e Ambiental. Campina Grande, v.19, n.5, p.418-425, 2015.

SILVA, R. O.; SOUSA, A. T. Caracterização de voçorocas em Caldas Novas (GO). In: II CONGRESSO DE ENSINO, PESQUISA E EXTENSÃO DA UEG, 2015, Pirenópolis. Anais eletrônicos. Pirenópolis: UEG, 2015.

SOUZA, W. R S. SILVA, S. A. S. SILVA, R. M da, ALVES, R. F., SILVA, J R. Atributos físico-químicos do solo em uma área de voçoroca no município de Brasil Novo - Pará: um estudo de caso. Revista Tree dimensional, Pro Floresta Goiânia, v.3 n.5. 2018. 14p.

YADA, M. M., MINGOTTE. F. L. C., MELO, W. J. de, MELO, G. P. de, MELO V. P. de, LONGO, R. M. RIBEIRO, A. I. R. Atributos Químicos e Bioquímicos em Solos Degradados por Mineração de Estanho e em Fase de Recuperação em Ecossistema Amazônico, Revista Brasileira de Ciencias do solo, São Paulo, 2015,724p. 